Background Total knee and hip arthroplasties are one of the most common and costly surgical procedures. They are performed to relieve pain and improve the patient's quality of life.

Purpose To describe the use of prostheses in hip and knee replacement surgery in a 1200-bed hospital.

Materials and Methods Descriptive retrospective study of the prostheses used in elective total hip and knee arthroplasties during year 2011.

Surgical orthopaedic interventions records and clinical histories were reviewed. Variables studied: sex, age, number of total hip and knee replacements performed: primary and revision (prosthetic replacement) procedures and reasons for revision surgery.

Results 94 total hip arthroplasties were carried out: 80 primary surgical procedures (85.1\%) performed on 40 men and 40 women, with a median age of 64 years (20-84), and 14 revision surgical procedures $(14.9 \%)$ performed on 4 men and 10 women, with a median age of 75 years (46-84). 2 of these patients had undergone primary surgery in the same year.

Reasons for prosthetic replacement were: aseptic loosening: 6 patients $(6.4 \%)$, dislocation: $4(4.3 \%)$, pain: $3(3.2 \%)$ and infection: $(1.1 \%)$.

140 total knee replacement procedures were carried out: 125 primary procedures (89.29\%) performed on 28 men and 99 women, with a median age of 71 years (42-87), and 15 revision procedures (10.71\%) performed on 2 men and 13 women, with a median age of 72 years $(65-80)$.

Etiologic factors of revision were: stiffness: 5 patients $(3.6 \%)$, instability: $5(3.6 \%)$, pain: $2(1.4 \%)$, aseptic loosening of the prosthesis: $2(1.4 \%)$ and infection: $1(0.7 \%)$.

Conclusions In most cases, both procedures are performed in patients younger than 75 years in order to improve their quality of life.

Total knee replacement surgery is more common than hip replacement. It is mainly performed in women and revision surgical procedures are less likely.

Prosthetic infection is the most important complication after surgery, but fortunately, is the least frequent cause of revision surgery.

No conflict of interest.

\section{OHP-044 HOW FAMILIAR ARE JOB ROLES OF HOSPITAL PHARMACISTS TO PHARMACY STUDENTS?}

doi:10.1136/ejhpharm-2013-000276.418

${ }^{1} \mathrm{M}$ Tomic, ${ }^{2} \mathrm{G}$ Jevtic, ${ }^{2} \mathrm{D}$ Rajinac, ${ }^{2} \mathrm{M}$ Kara-Jovanovic, ${ }^{2} \mathrm{LJ}$ Stojicevic, ${ }^{2} \mathrm{M}$ Klancnik. 'Clinical Centre of Serbia, Service for the pharmaceutical business and supply, Belgrade, Serbia; ${ }^{2}$ Clinical Centre of Serbia, Emergency Centre, Belgrade, Serbia

Background Pharmacists in a Clinical Centre in Serbia are involved in various educational programmes for pharmacy students.

Purpose To evaluate how much information pharmacy students had about the activities of pharmacists in hospitals.

Materials and Methods A survey containing 32 questions has been conducted among the 58 students of both genders, varying interests and academic achievement in the final year of study. $75 \%$ of questions were multiple-choice and the rest were related to specific cases; opinions and suggestions were requested as well.

Results 35 of the 58 respondents thought that pharmacists didn't participate in public procurement and 22 thought that pharmacists didn't participate in the supply of medical devices. 39 thought that a pharmacist made a decision on the use of the appropriate drug from a particular pharmacotherapy group, 46 thought that the hospital pharmacist decided on the posology of the appropriate drug, while 56 thought that pharmacists were regularly consulted by the medical staff on the dissolution of certain medicines (antibiotics and cytostatics). The same number also had an opinion that pharmacists were always consulted about drug interactions. $64 \%$ of students believed that they had sufficient knowledge of chemistry, pharmaceutical technology and pharmacotherapy, but insufficient knowledge in certain medical areas - anatomy, pathology and physiology. $78 \%$ of students thought that basics of hospital pharmacy should be introduced as an optional subject during undergraduate studies or there should be appropriate specialisation in this field after graduation.

Conclusions More than half of the students were not sufficiently informed about hospital pharmacy, but they were eager to learn things that would help them in their future practise. It suggests that fellow practitioners should be actively engaged in continuing education programmes for students, and developing better cooperation with the faculty of pharmacy in order to provide both theoretical and practical knowledge in the field of hospital pharmacy.

No conflict of interest.

\section{OHP-045 IMPACT OF A MULTIDISCIPLINARY STAFF MEETING ON ANTIBIOTIC TREATMENT OUALITY FOR OSTEOARTICULAR INFECTIONS IN AN ORTHOPAEDIC SURGERY CARE UNIT}

doi:10.1136/ejhpharm-2013-000276.419

'S Bauer, ${ }^{2} \mathrm{MA}$ Bouldouyre, ${ }^{2} \mathrm{~A}$ Oufella, ${ }^{3 \mathrm{P}}$ Palmari, ${ }^{4} \mathrm{R}$ Bakir, ${ }^{4} \mathrm{H}$ Gros, ${ }^{2} \mathrm{~A}$ Fabreguettes ${ }^{1}$ Centre Hospitalier General de Perpignan, Pharmacy, Perpignan Cedex 9, France, ${ }^{2}$ Centre Hospitalier Intercommunal Robert Ballanger, Pharmacy, Aulnay Sous Bois, France; ${ }^{3}$ Centre Hospitalier Intercommunal Robert Ballanger, Orthopaedic Surgery Care Unit, Aulnay Sous Bois, France; ${ }^{4}$ Centre Hospitalier Intercommunal Robert Ballanger, Medecine And Infectious Desease Care Unit, Aulnay Sous Bois, France

Background Treating osteoarticular infections is difficult

Purpose To evaluate professional practise, we studied the effect of a multidisciplinary staff meeting on the quality of antibiotic treatment in an orthopaedic surgery care unit.

Materials and Methods Via the coding process, we retrospectively studied patients hospitalised for osteoarticular infections (diabetic foot excluded) in the orthopaedic care unit of a general hospital in France. We compared antibiotic treatment conformity to good practise (bacteriology, dose, length of treatment, time taken to implementing microbiology report), length of hospitalisation and 6 month-outcome, for patients with osteoarticular infections, before (March 2007 to March 2009) and after (March 2009 to March 2011).implementation of the multidisciplinary staff meeting.

Results 85 patients were selected and 77 files were examined. Fifty-five medical records were actively devoted to osteoarticular infection and all of them were analysed: this worked out at 30 patients (32 infections) before the staff meetings and 26 patients (28 infections) after the staff meetings had started. Staff meeting decisions were reported in medical files in $72 \%$ of cases. Before staff meetings were instituted, antibiotic treatment was changed in $47 \%$ of cases, versus $96 \%$ since establishment of the staff meeting ( $p<0.0001)$. Dose was optimum in $72 \%$ of infections before staff meetings were instituted, versus $89 \%$ afterwards $(\mathrm{P}=0.11)$ and length of antibiotic treatment conformed to recommendations in $41 \%$ of infections before staff meetings, versus $86 \%$ after staff meetings had begun $(\mathrm{P}=0,0005)$. The average time to respond to an antibiogram decreased from 2 days before staff meetings to 1.7 days after staff meeting $(\mathrm{P}=0.43)$, and length of hospitalisation was 19.8 days before staff meetings versus 23.1 days after $(\mathrm{P}=0.49)$. Recovery at 6 months accounted for $62 \%$ of patients before staff meetings, versus $76 \%$ after staff meetings $(P=0.35)$ and failure at 6 months concerned $29 \%$ of infections before staff meetings versus $24 \%$ after their institution $(\mathrm{P}=0.75)$.

Conclusions Since the beginning of multidisciplinary staff meeting in our orthopaedic surgery care unit, antibiotic treatment has significantly improved concerning spectrum and duration of 
treatment $(\mathrm{p} \leq 0.0005)$. With this limited sample, clinical impact at 6 months was not significant.

No conflict of interest.

\section{OHP-046 IMPACT OF MULTI-LUMEN INFUSION DEVICES ON THE OCCURRENCE OF PHYSICAL DRUG INCOMPATIBILITIES: A CONTROLLED IN VITRO STUDY}

doi:10.1136/ejhpharm-2013-000276.420

'M Perez, ' ${ }^{1} \mathrm{~A}$ Foinard, ${ }^{1} \mathrm{C}$ Barthélémy, ${ }^{2} \mathrm{~B}$ Debaene, 'B Décaudin, 'P Odou. 'Faculté de Pharmacie, Laboratoire de Biopharmacie Pharmacie Galénique et Hospitalière, Lille Cedex, France; ${ }^{2} \mathrm{CHU}$ de Poitiers, Département d'Anesthésie, Poitiers, France

Background Drug incompatibility is a problem when managing patients in intensive care units. Patients receive many drugs simultaneously but through limited venous accesses. The recent marketing of new multi-lumen infusion access device may open the way to preventing incompatibility.

Purpose To evaluate the impact of multi-lumen infusion access devices connected to single-lumen central venous catheters on the occurrence of known drug incompatibilities through a controlled in vitro study.

Materials and Methods Two infusion devices were studied: 1) a standard set with six-gang-manifolds and its extension line and 2) a multi-lumen infusion access device with nine lumens (Edelvaiss-Multiline, Doran International, France). Six drugs were selected: three basic drugs (furosemide, pantoprazole and amoxicillin/clavulanic acid) and three acid drugs (amiodarone, dobutamine and midazolam). Two, four or six drugs and an infusion vehicle (saline, Ringer's or 5\% glucose) were infused simultaneously. The infusion rate of the vehicle was initially set at $100 \mathrm{~mL} / \mathrm{h}$ and decreased stepwise by $10 \mathrm{~mL} / \mathrm{h}$ until precipitate formation occurred. Physical incompatibility was assessed by visual inspection and sub-visible particle count test as defined by the European Pharmacopeia according to the European Pharmacopeia. The lowest value of the vehicle infusion rate that satisfied the two tests was reported for each infusion set and for each drug combination.

Results The use of multiline access devices contributed to preventing drug incompatibilities when simultaneously infusing two and four drugs. Indeed, infusion vehicle flow rate gains oscillated between 10 and $40 \mathrm{~mL}$ per hour, in more than $55 \%$ and $25 \%$ of cases, respectively for two and four drugs. When infusing six drugs simultaneously, no differences were identified.

Conclusions Our main hypothesis is that fluid dynamics differ depending on the infusion device resulting in differences in the contact time between drugs. Under specified infusion conditions, the nine-lumen device prevents physical drug incompatibilities.

No conflict of interest.

\section{OHP-047 IMPACT OF THE ECONOMIC CRISIS ON BIOMEDICAL RESEARCH: ANALYSIS OF THE WORK OF A CLINICAL RESEARCH ETHICS COMMITTEE}

doi:10.1136/ejhpharm-2013-000276.421

P Arcenillas, S Redondo, N Giménez, P March, L Soriano, R Pla, S Quintana. Hospital Universitari Mútua de Terrassa, Pharmacy, Terrassa, Spain

Background Since 2008, the economic crisis has directly affected many activities, health and biomedical research being particular fields involved.

Purpose To evaluate the impact of the economic crisis on research at a Spanish hospital based on an analysis on the work done by the Clinical Research Ethics Committee (CEIC). To compare it with any effects on Spanish and European CTs.
Materials and Methods CEIC Minutes from a 500-bed university hospital were reviewed from 2000 to 2011, obtaining information from clinical trials (CTs) and observational studies (OS).

The financing of CTs was classified: 1) CTs promoted by the pharmaceutical industry, 2) by scientific societies with industry support, 3) by scientific societies with government support and 4) unfunded CTs. We compared two periods: pre-crisis (2000-2007) and crisis (2008-2011).

National scientific activity was obtained from a secondary data source from the information provided by the Agencia Española de Medicamentos y Productos Sanitarios and the European activity from EU Clinical Trials Registers (European Medicines Agency website).

Data analysis used conventional descriptive statistics.

Results 782 protocols were evaluated (average 71 protocols/year).

During 2008-2011 there was an annual average decrease of 13 CT in groups 1 and 2, compared with the period 2000-2007 (95\% CI: 4-22 CT).

Regarding the OSs, there was an annual average increase of 36 OSs during the second period (95\% CI: $24-49$ OS). There were no statistical differences between the two periods for groups 3 and 4.

The total number of protocols increased by an average of 25 projects/year during the second period compared to the first $(95 \% \mathrm{CI}$ : $8-40$ projects).

There were 2340 CTs in Spain during the first period and 3096 during the second $(p=n s)$. CTs in Europe were 7,908 and 10,632 respectively $(p=n s)$.

Conclusions The CEIC workload was maintained, even increased, but because of OSs and unfunded research. The crisis marked a turning point; funded studies decreased and OSs increased.

At the moment there are no noteworthy changes in Spanish or European CT activity.

Abstract OHP-047 Table 1

\begin{tabular}{lll}
\hline & Number of CTs & $\%$ \\
\hline Group 1 & 407 & 52 \\
Group 2 & 53 & 7 \\
Group 3 & 32 & 4 \\
Group 4 & 93 & 12 \\
OS & 197 & 25 \\
\hline * OS were analysed globally
\end{tabular}

No conflict of interest.

\section{OHP-048 IMPLEMENTATION OF A PROTOCOL FOR SELECTION OF BIOLOGICAL THERAPIES IN RHEUMATOLOGY}

doi:10.1136/ejhpharm-2013-000276.422

YM Alonso-Triana, H Navarro, R Huarte, I Larrodé, M Sebastián-Aldeanueva, MJ Perrino, R Abad. Servet, Pharmacy, Zaragoza, Spain

Background The Public Health System in our Autonomous Community has established a protocol for biological treatments (BTs) in rheumatoid arthritis (RA), spondyloarthropathies (SAPs) and juvenile idiopathic arthritis (JIA).

Purpose To evaluate the implementation of the BTs protocol and to analyse the use of these treatments.

Materials and Methods We analysed patients who had started treatment with BT or been switched from a previous biological treatment, since the implementation of the protocol (12/05/2011 to 29/02/2012). This document has different levels of decision based on both disease status and treatment effectiveness; RA: 1st level: infliximab or subcutaneous tumour necrosis factor inhibitor (anti-TNF) (etanercept or adalimumab); 2nd: tocilizumab or abatacept or rituximab; 3rd: golimumab or certolizumab pegol. SAPs: 1st level: infliximab or etanercept or adalimumab; 2 nd: golimumab; 3 rd: infliximab 